\title{
A virtual cycle: theory and experiment converge on the exit from mitosis
}

\author{
Karl A Merrick ${ }^{1,2}$ and Robert P Fisher ${ }^{1 *}$
}

Addresses: ${ }^{1}$ Department of Structural and Chemical Biology, Mount Sinai School of Medicine, 1 Gustave L Levy Place, Box 1677, New York, NY 10029, USA; ${ }^{2}$ Programs in Biochemistry, Cell and Molecular Biology, Weill Cornell Graduate School of Medical Sciences, New York, NY 10065, USA

* Corresponding author: Robert P Fisher (robert.fisher@mssm.edu)

FI000 Biology Reports 2010, 2:33 (doi:10.34I0/B2-33)

The electronic version of this article is the complete one and can be found at: http://fl000.com/reports/biology/content/2/33

\begin{abstract}
The cell division cycle can be modelled as a series of quantitative thresholds of cyclin-dependent kinase (CDK) activity. DNA synthesis has a lower threshold requirement for CDK than does entry into mitosis, and mitotic exit and re-setting of replication origins occur upon collapse of CDK activity below both thresholds, so that the simple rise and fall of CDK with each cell cycle might suffice to ensure repeated alternation of chromosome duplication and segregation. Recent experimental dissections of mitotic exit, which have both guided and been informed by computational modelling, suggest a more complicated mechanism, in which unidirectional progression is ensured by systemslevel control of CDK function and the balance between mitotic CDK and phosphatase activities.
\end{abstract}

\section{Introduction and context}

Eukaryotic cells enter mitosis with high levels of active cyclin-dependent kinase (CDK), which phosphorylates numerous proteins, leading to an array of cellular changes [1]. High kinase levels are also important for maintaining the mitotic state; inhibition of CDK in cells arrested in mitosis with spindle poisons causes aberrant progression into interphase [2-4]. At anaphase onset, the anaphase-promoting complex (APC) becomes active and polyubiquitinylates proteins to target them for degradation. Among the proteins tagged for destruction is cyclin, with consequent lowering of mitotic CDK (Cdk1) activity. Supraphysiologic levels of a non-degradable cyclin can prevent mitotic exit, which led to the hypothesis that cyclin degradation is necessary and sufficient to drive cells out of mitosis $[1,5]$.

In Saccharomyces cerevisiae, mitotic exit also depends on the phosphatase Cdc14, which dephosphorylates many Cdk1 substrates. Cdc14 is sequestered by the Net1 protein in the nucleolus until anaphase, when it is released by the sequential action of the Cdc14 early anaphase release (FEAR) and mitotic exit network pathways [6]. In metazoans, as in yeast, dephosphorylation of mitotic substrates is a hallmark of the M/G1 transition [7], but the responsible phosphatases are only now beginning to emerge.

\section{Major recent advances}

A central prediction of the simple CDK threshold model was that mitotic exit would be effectively inhibited by levels of Cdk1 activity normally attained during metaphase [8]. To test this, Cross and colleagues [9] developed a system that allowed precisely controlled expression of a fluorescently labelled, non-degradable mitotic cyclin, Clb2, in budding yeast. Surprisingly, Clb2 expression sustained at the peak level reached during a normal mitosis delayed but did not prevent the events of mitotic exit. Because Cdc14 was released from the nucleolus with normal efficiency and kinetics in cells expressing non-degradable Clb2, the authors hypothesized that mitotic exit is controlled by the ratio of Cdk1 to Cdc14, not by simply crossing a threshold of Cdk1 activity. A mathematical model incorporating this additional complexity predicted experimental results of 
expressing non-degradable Clb2 or high levels of Sic1, a CDK inhibitor involved in mitotic exit, more accurately than did the simple threshold model [9].

Although cells expressing non-degradable Clb2 could exit mitosis, they showed abnormalities during the following cycle in the response to mating factor and in nucleation of the metaphase spindle [9]. This suggested that the persistence of stable Cdk1-Clb2 complexes might disturb the smoothly unidirectional progression of the cell cycle even when mitotic exit per se could be accomplished. Irreversibility of mitotic exit was widely assumed to be the inevitable result of mitotic cyclin destruction, but experiments by Uhlmann and colleagues [4] exposed the logical flaw in this reasoning (which had been pointed out by Novak, Tyson, and colleagues [10]) by showing that irreversibility of Cdk1 inhibition and mitotic exit are instead a consequence of systems-level feedback. Also working with budding yeast, they asked if destruction of Clb2 was sufficient to drive irreversible mitotic exit. Within about 50 minutes after release from a metaphase block, Clb2 was degraded almost completely, mitotic phosphoproteins became dephosphorylated, and mitotic spindles broke down. Nevertheless, if the APC was then inactivated, these events could be reversed (Figure 1a). Their interpretation: Clb2 destruction is not sufficient for irreversible mitotic exit because its degradation can be balanced by re-synthesis, which, like proteolysis, is a thermodynamically irreversible process [4].

When the APC was allowed to remain active for just 10 minutes longer, mitotic exit became essentially irreversible. This correlated with the accumulation of Sic1 and, in sic1 $\Delta$ cells, mitotic exit remained reversible for up to 90 minutes. This behavior was simulated by a mathematical model; irreversibility occurred once Sic1 accumulated to a threshold level, whereas below this threshold Clb2 re-synthesis could drive cells back into a mitotic state. Expression and stability of Sic1 are regulated by the balance of Cdk1 and Cdc14 activity, suggesting that the switch for mitotic exit proposed by Cross and colleagues might be built to ensure irreversibility (Figure 1b).

\section{Future directions}

Less is known about the control of mitotic exit in higher eukaryotes, but several studies suggest that its basic regulatory logic is conserved. Gorbsky and colleagues $[2,11]$ reported that mitotic exit caused by chemical inhibition of Cdk1 was fully reversible if mitotic cyclin degradation was prevented in cultured Xenopus and human cells. As in budding yeast, there is a finite window in which reversal can occur. This interval of reversibility appears to end with activation of the kinases that phosphorylate Cdk1 to inhibit its activity, Wee1 and Myt1 [11], which are themselves antagonized by Cdk1 in a double-negative feedback loop [1]. Modelling suggests that this loop could function analogously to the one comprising Cdk1 and its antagonists in yeast to prevent mitotic re-entry [12]. Some caution in accepting these conclusions is still warranted, however, because of another study in which mitotic exit did not occur in human cells when Cdk1 and the proteasome were simultaneously inhibited [3].

The disparate results reported by the Gorbsky and Margolis groups $[2,3,11]$ have yet to be reconciled. There is general agreement, however, that inhibiting Cdk1 with small molecules can trigger mitotic exit in the absence of spindle function in cells with working APCs and proteasomes. In the same paper that questioned the occurrence of mitotic exit in the absence of proteasome activity, Margolis and colleagues [3] found another way to block exit triggered by Cdk1 inhibition - addition of okadaic acid, an inhibitor of protein phosphatases PP1 and PP2A. Kornbluth and colleagues [13] later reported that PP1 is the major activity responsible for dephosphorylating mitotic phosphoproteins in Xenopus egg extracts and HeLa cells. Cdk1 phosphorylates and inhibits PP1, and the degradation of mitotic cyclin partially relieves that inhibition to allow PP1 autodephosphorylation and subsequent inactivation of a PP1-inhibitory protein. This is reminiscent of the regulation of Cdc14 and its fission yeast ortholog Clp1, in that the phosphatase participates in a positive feedback loop to ensure its own robust activation during mitotic exit $[6,14]$. There appears to be something missing from the metazoan circuitry, however. In S. cerevisiae, high Cdk1 levels at anaphase initially promote $\mathrm{Cdc} 14$ release by phosphorylating Net1 (Figure 1b), whereas in metazoans Cdk1 activity must decrease before PP1 becomes active. This difference raises two possibilities: (a) the model of Drapkin et al. [9] (in which the CDK:phosphatase ratio is the determining factor for mitotic exit) is incompletely conserved in metazoans; or (b) there remains to be discovered an additional phosphatase promoting mitotic exit in metazoans, which can become active before mitotic cyclin is destroyed.

PP2A is a candidate to be the 'missing' phosphatase $a$ priori because of its known sensitivity to okadaic acid, which, as mentioned above, can block mitotic exit. Moreover, Hunt and colleagues [15] recently demonstrated a role for PP2A in promoting mitotic exit in Xenopus egg extracts. They detected a phosphatase that was regulated during the cell cycle and active towards a 
(a)

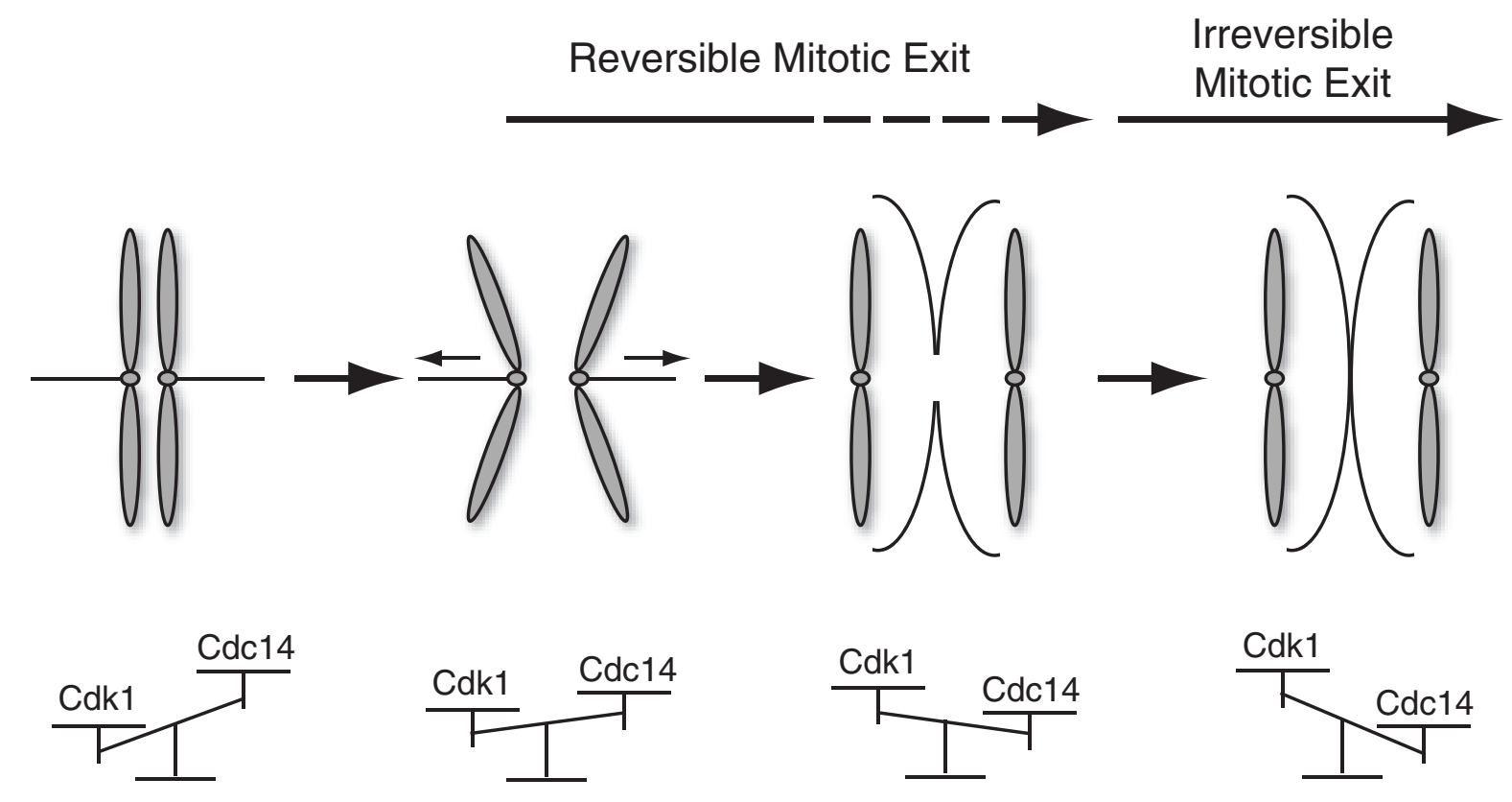

(b)

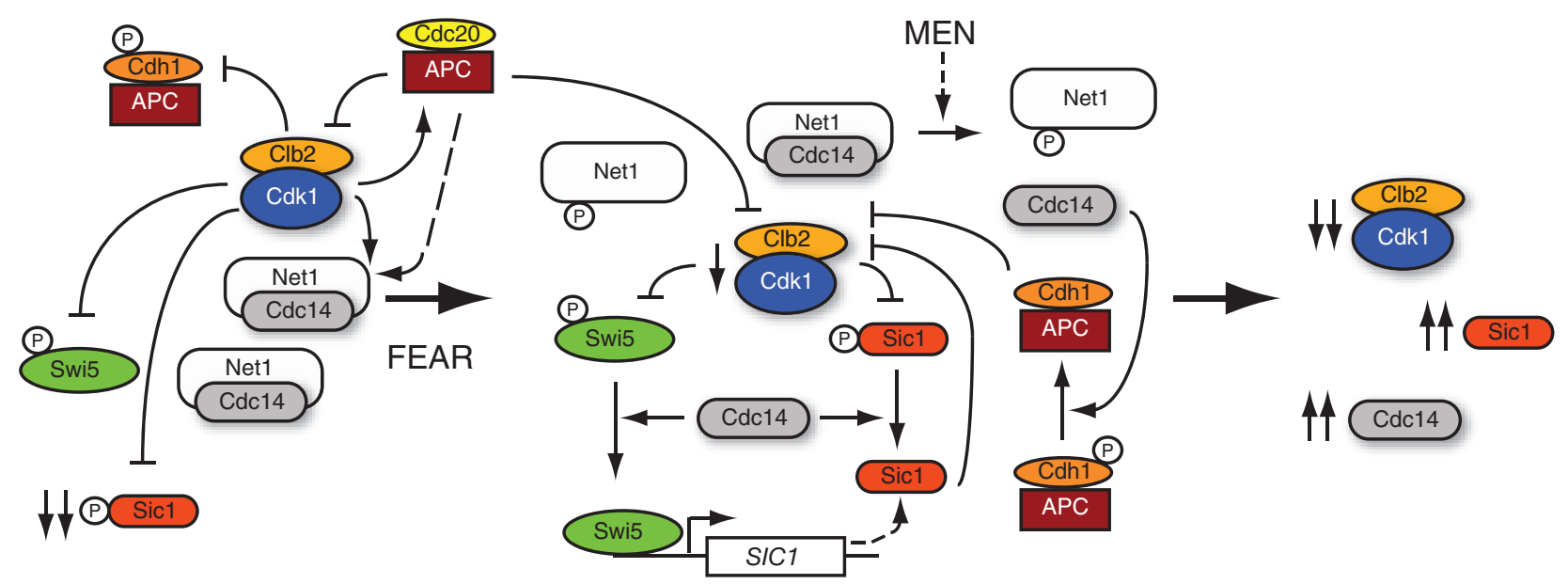

(a) A balance between Cdkl and Cdcl4 activities governs mitotic exit in budding yeast. Early in mitosis, Cdkl activity is at its peak while Cdcl4 activity is very low. As cells progress through anaphase, Cdkl activity drops and Cdcl4 activity rises to trigger mitotic exit. This transition is reversible despite mitotic cyclin degradation - until Sicl protein accumulates beyond a threshold level [discussed in (b)]. (b) The circuitry controlling an irreversible switch. Early in mitosis, phosphorylation by $\mathrm{CdkI}-\mathrm{Clb}$ leads to downregulation of the Cdh I-activated form of the anaphase-promoting complex (APC), the transcription factor Swi5, and the cyclin-dependent kinase (CDK) inhibitor Sicl. As cells enter anaphase, CdkI phosphorylates Netl, triggering an initial, partial release of Cdcl4 by the Cdcl4 early anaphase release (FEAR) pathway (which also requires APC function). Concomitantly, the Cdc20-bound form of the APC (which is positively regulated by CDK activity) ubiquitylates mitotic cyclins to target them for degradation. Later in anaphase, the remaining Cdcl4 is released due to activation of the mitotic exit network (MEN) pathway, allowing for more extensive dephosphorylation of mitotic phosphoproteins. Unphosphorylated Swi5 can trigger transcription and accumulation of Sicl, which is now able to evade phosphorylation-directed ubiquitinylation and degradation, leading to further CdkI inhibition. Activation of APC-CdhI leads to further destruction of mitotic cyclins as cells complete exit from mitosis. It is through this system of feedback loops that irreversibility of mitotic exit is ensured. 
CDK substrate, and identified it as PP2A associated with the B55 $\delta$ regulatory subunit. Immunodepletion of B55 $\delta$ from extracts accelerated entry to, and prevented exit from, mitosis. Interestingly, depletion of PP2A/B55 $\delta$ was effective at delaying mitotic entry only when performed on an interphase extract (i.e., prior to mitotic entry); depletion of the phosphatase from extracts already in mitosis had little or no effect on the rate of dephosphorylation of an APC subunit phosphorylated by CDK. This implies that other phosphatases (such as PP1) can dephosphorylate the bulk of mitotic phosphoproteins, and that PP2A/B55 $\delta$ must execute its required function earlier, either in interphase before mitotic CDK activation or at mitotic entry [15]. However, activity of this PP2A isoform is abruptly downregulated upon entry to mitosis, which might argue against a role strictly analogous to that of the 'early release' form of Cdc14 in budding yeast.

Here we have summarized recent advances in understanding mitotic exit and in building mathematical models that accurately predict behavior of the mitotic control system in vivo. These studies have made it clear that features such as irreversibility are the product of systems-level feedback, not the thermodynamic irreversibility of any one reaction - a generalization likely to hold true throughout evolution [10]. It is also possible that yeast-like feedback loops containing CDKs and opposing phosphatases make mitotic exit robust and switch-like in metazoan cells. Before we can be certain of that conclusion, however, more of the relevant phosphatases probably need to be identified, their roles must be more precisely defined, and the regulation of their localization and activity will need to be elucidated.

\section{Abbreviations}

APC, anaphase-promoting complex; CDK, cyclindependent kinase.

\section{Competing interests}

The authors declare that they have no competing interests.

\section{Acknowledgements}

We thank members of the Fisher lab for helpful discussions. Work in the lab is supported by National Institutes of Health grants GM056985 and GM076021.

\section{References}

I. Morgan DO: The Cell Cycle: Principles of Control. London: New Science Press Ltd; 2007.

2. Potapova TA, Daum JR, Pittman BD, Hudson JR, Jones TN, Satinover DL, Stukenberg PT, Gorbsky GJ: The reversibility of mitotic exit in vertebrate cells. Nature 2006, 440:954-8.

FI000 Factor 8.2 Exceptional

Evaluated by Tom Misteli 21 Apr 2006, Linda Wordeman 21 Apr 2006, Claire Walczak 24 Apr 2006, Kerry Bloom 26 Apr 2006

3. Skoufias DA, Indorato RL, Lacroix F, Panopoulos A, Margolis RL: Mitosis persists in the absence of Cdkl activity when proteolysis or protein phosphatase activity is suppressed. J Cell Biol 2007, 179:671-85.

4. Lopez-Aviles S, Kapuy O, Novak B, Uhlmann F: Irreversibility of mitotic exit is the consequence of systems-level feedback. Nature 2009, 459:592-5.

FI000 Factor 6.0 Must Read Evaluated by Robert Fisher 02 Jun 2009

5. Wasch R, Cross FR: APC-dependent proteolysis of the mitotic cyclin Clb2 is essential for mitotic exit. Nature 2002, 4I 8:556-62.

6. Queralt E, Uhlmann F: Cdk-counteracting phosphatases unlock mitotic exit. Curr Opin Cell Biol 2008, 20:66I-8.

7. Olsen JV, Vermeulen M, Santamaria A, Kumar C, Miller ML, Jensen LJ, Gnad F, Cox J, Jensen TS, Nigg EA, Brunak S, Mann M: Quantitative phosphoproteomics reveals widespread full phosphorylation site occupancy during mitosis. Sci Signal 2010, 3:ra3.

8. Chen KC, Calzone L, Csikasz-Nagy A, Cross FR, Novak B, Tyson J]: Integrative analysis of cell cycle control in budding yeast. Mol Biol Cell 2004, I 5:384I-62.

FI000 Factor 6.4 Must Read

Evaluated by J Julian Blow 25 Aug 2004, Jacky Snoep 28 Oct 2004

9. Drapkin BJ, Lu Y, Procko AL, Timney BL, Cross FR: Analysis of the mitotic exit control system using locked levels of stable mitotic cyclin. Mol Syst Biol 2009, 5:328.

I0. Novak B, Tyson J], Gyorffy B, Csikasz-Nagy A: Irreversible cellcycle transitions are due to systems-level feedback. Nat Cell Biol 2007, 9:724-8.

II. Potapova TA, Daum JR, Byrd KS, Gorbsky GJ: Fine tuning the cell cycle: activation of the Cdkl inhibitory phosphorylation pathway during mitotic exit. Mol Biol Cell 2009, 20:1737-48.

12. Kapuy O, He E, Uhlmann F, Novak B: Mitotic exit in mammalian cells. Mol Syst Biol 2009, 5:324.

13. Wu JQ, Guo JY, Tang W, Yang CS, Freel CD, Chen C, Nairn AC, Kornbluth S: PPI-mediated dephosphorylation of phosphoproteins at mitotic exit is controlled by inhibitor-I and PPI phosphorylation. Nat Cell Biol 2009, I I:644-5I.

FI000 Factor 3.0 Recommended

Evaluated by Brian Wadzinski OI Jun 2009

14. Wolfe BA, McDonald WH, Yates JR 3rd, Gould KL: Phosphoregulation of the $\mathrm{Cdc} \mathrm{I4/Clp} \mathrm{I} \mathrm{phosphatase} \mathrm{delays} \mathrm{late} \mathrm{mitotic}$ events in S. pombe. Dev Cell 2006, I I:423-30.

FI000 Factor 3.0 Recommended

Evaluated by Stephen Doxsey 22 Sep 2006

I5. Mochida S, Ikeo S, Gannon J, Hunt T: Regulated activity of PP2AB55 delta is crucial for controlling entry into and exit from mitosis in Xenopus egg extracts. EMBO J 2009, 28:2777-85. 